\title{
BUOYANCY-INDUCED FLOW AND HEAT TRANSFER OF POWER LAW FLUIDS IN A SIDE HEATED SQUARE CAVITY
}

\author{
Ternik, P.* \& Buchmeister, J.** \\ *Ternik Primož - Private Researcher, Bresterniska 163, 2354 Bresternica, Slovenia \\ ${ }^{* *}$ University of Ljubljana, Faculty of Pharmacy, Askerceva 7, 1000 Ljubljana, Slovenia \\ E-Mail: pternik@pt-rtd.eu, julija.buchmeister@gmail.com
}

\begin{abstract}
This paper details the numerical study of laminar natural convection in a square enclosure filled with a non-Newtonian fluid. Thermal boundary conditions of the Dirichlet type are applied on the vertical walls of the enclosure while the horizontal ones are assumed adiabatic. A Power-law model is used to characterize the viscous behaviour of the purely viscous non-Newtonian fluids. The governing differential equations have been solved by the standard finite volume method and the hydrodynamic and thermal fields were coupled together using the Boussinesq approximation.

The effects of Power-law index $(n)$ in the range $0.50 \leq n \leq 1.50$ on the heat and momentum transport are investigated for the values of Rayleigh number $(R a)$ in the range $10^{1} \leq R a \leq 10^{4}$ and a Prandtl number of $\operatorname{Pr}=10$.

We report accurate results of a systematic study with a focus on the most important buoyancyinduced flow and heat transfer characteristics. It is shown that the mean Nusselt number values increases with the increasing values of Rayleigh number for Newtonian as well as Power-law fluids. However, shear-thickening fluids $(n>1)$ are characterised with smaller $\overline{N u}$ values. Finally, the onset of convection dominated heat transfer mechanism is shifted towards lower values of $R a$ for the shearthinning fluids $(n<1)$.

(Received in April 2014, accepted in January 2015. This paper was with the authors 4 months for 2 revisions.)
\end{abstract}

Key Words: Laminar Natural Convection, Differentially Heated Cavity, Mean Nusselt Number, Critical Rayleigh Number, Numerical Modelling

\section{INTRODUCTION}

Today more than ever, controlled heat transfer plays an important role in the development of energy-efficient heat transfer systems and fluids which are required in many industries and commercial applications [1-4]. Natural convection (i.e. flow caused by the temperature induced density variations) is one of the most extensively analysed heat transfer configurations because of its fundamental importance as the "benchmark" problem for studying convection effects (and comparing as well as validating numerical techniques). For example, an accurate benchmark solution for natural convection of air in a square cavity with vertical boundaries kept at different temperatures are presented in [5], with reference to $10^{3} \leq R a \leq 10^{6}$. Another benchmark solution for natural convection of air in a side heated square cavity is reported in [6] for $10^{4} \leq R a \leq 10^{6}$. Finally, Nonino and Croce [7] have extended the results presented in $[5,6]$ to the case of higher values of Rayleigh number; i.e $10^{5} \leq R a \leq 10^{8}$.

In spite of geometrical simplicity, buoyancy driven flows are complex because of essential coupling between the transport properties of flow and thermal fields. In particular, internal flow problems are considerably more complex than external ones. This is because at large Rayleigh number classical boundary layer theory yields the simplifications for external flow problems, namely, the region exterior to the boundary layer is unaffected by the boundary layer. For confined natural convection, in contrast, boundary layers form near the walls but the region exterior to them in enclosed by the boundary layers form a core region. Since the core is partially or fully encircled by the boundary layers, the core flow is not 
readily determined from the boundary conditions but depend on the boundary layer, which, in turn is influenced by the core. The interactions between the boundary layer and core constitute a major complexity in the problem. In fact, the situation is even more intricate because it often appears that more than one global core flow is possible and flow sub-regions, such as, cells and layers, may be embedded in the core.

In addition to the obvious academic interest and flow richness, this configuration has occupied the centre stage in many fundamental heat transfer analysis which is of prime importance in various technological applications [8, 9]. Although quite some various configurations of the enclosure problem are possible $[10,11]$, one of the most studied cases is the two-dimensional square enclosure with differentially heated isothermal vertical walls and adiabatic horizontal walls. Such a configuration is one of the most extensively studied configuration for Newtonian flows. On the other hand, a range of materials encountered in an engineering practice display a complex behaviour and this is generally due to their large molecular structure. As a consequence, the viscosity of these fluids may depend on the deformation rate and some of them also show elastic behaviour, i.e. they combine the characteristics of a solid and a fluid. Such materials can be conveniently grouped into three general classes:

- Fluids for which the shear stress at any point is determined only by the rate of shear; these fluids are variously known as 'time independent', 'purely viscous', 'inelastic' or 'generalised Newtonian fluids' (GNF).

- More complex fluids for which the relation between shear stress and shear rate depends, in addition, upon the duration of shearing and their kinematic history; they are called 'timedependent fluids'.

- Substances exhibiting characteristics of both ideal fluids and elastic solids and showing partial elastic recovery after deformation; these are categorized as 'viscoelastic fluids'.

Regarding the generalized Newtonian fluids, the most common type of viscous behaviour observed is shear-thinning, characterized by the viscosity which decreases with the increasing shear rate. Although the Power-law model offers the simplest representation of purely viscous behaviour [12], it is perhaps the most widely used rheological model in the literature dealing with the process engineering applications. Table I provides a compilation of the Power-law constants for rheological modifiers (which are present in the cosmetic formulation to give a product with the desired viscosity) as well as cosmetic (i.e. personal care) products.

Table I: Typical values of Power-law constants.

\begin{tabular}{|l|c|c|}
\hline \multicolumn{1}{|c|}{ Rheological modifier [13] } & $\boldsymbol{k}\left(\boldsymbol{P a} \boldsymbol{s}^{\boldsymbol{n}}\right)$ & $\boldsymbol{n}(-)$ \\
\hline Liporamnosan $1.8 \%$ & $4.744 \times 10^{-3}$ & 0.67 \\
\hline Natrosol 250 MR 2.0\% & $1.194 \times 10^{-3}$ & 0.87 \\
\hline Methocell M312 $0.9 \%$ & $9.915 \times 10^{-3}$ & 0.55 \\
\hline Lubrajel DV 2.3\% & $3.514 \times 10^{-3}$ & 0.33 \\
\hline Personal care product [14] & $\boldsymbol{k}\left(\boldsymbol{P a} \boldsymbol{s}^{\boldsymbol{n}}\right)$ & $\boldsymbol{n}(-)$ \\
\hline Nail polish & 750 & 0.86 \\
\hline Mascara & 200 & 0.24 \\
\hline Sunscreen lotion & 75 & 0.28 \\
\hline Oil of Olay & 25 & 0.22 \\
\hline
\end{tabular}

In comparison to the vast body of literature regarding the natural convection of Newtonian fluids, a comparatively limited effort has been directed towards understanding of buoyancy driven flow of non-Newtonian fluids in rectangular enclosures. Kim et. al [15] studied buoyant convection in a side heated square cavity filled with the shear-thinning $(n \leq 1.00)$ Power-law fluids. Their study concluded that the mean Nusselt number increases with 
decreasing Power-law index for a given range of values of Rayleigh $\left(R a=10^{5} \ldots 10^{7}\right)$ and Prandtl numbers $\left(\operatorname{Pr}=10^{2} \ldots 10^{4}\right)$. Lamsaadi et. al $[16,17]$ have studied the effect of the Power-law index in the high Prandtl number limit of tall and shallow enclosures where the side-wall boundary conditions are subjected to constant heat fluxes. Turan et al. [18] have studied steady natural convection of Power-law fluids in a square enclosure with differentially heated side walls with the Power-law index ranging from 0.60 to 1.80 for a range of values of Rayleigh and Prandtl number given by $R a=10^{3} \ldots 10^{6}$ and $\operatorname{Pr}=10^{1} \ldots 10^{5}$. Their simulation results show that the mean Nusselt number increases with increasing values of the Rayleigh number for both Newtonian and Power-law fuids. Furthermore, the Nusselt number was found to decrease with increasing Power-law index.

In the present work a steady and laminar buoyancy-induced flow of generalised Newtonian fluids in a differentially heated square enclosure has been studied by numerical means. A parametric study has been performed for a range of Rayleigh number values $10^{1} \leq R a \leq 10^{4}$, while the Power-law index values mapped the $0.50 \leq n \leq 1.50$ range, allowing for the investigation of both shear-thinning and shear- thickening effects at the conduction as well as convection dominated heat transfer regime.

\section{NUMERICAL METHOD}

The governing equations were solved by taking the advantage of the open-source OpenFOAM CFD software package which employs the standard finite volume method. It is written in $\mathrm{C}++$ and uses classes and templates to manipulate and operate scalars, vectorial and tensorial fields [19]. The advantages of using OpenFOAMas a CFD framework are that the software is freely available (with open source, licensed under GNU General License Software) and both flexible and highly extensible. Its hierarchical, open structure allows the user to make transparent modifications to the governing equations being solved, to tailor them to specific applications whilst retaining the benefits of a stable and general numerical framework.

Each governing equation is discretised in a space by integration over the set of control volumes forming the computational mesh. Such a process results in a system of linearized equations of mass, momentum and temperature conservation joined with the constitutive equation. In these equations all variables are evaluated (and stored) in the centre of control volumes populating the physical domain being considered.

The steady incompressible solver buoyantSimpleFoam (version 2.1.1) has been modified and used for the present study; governing equations were solved in a segregated manner, followed by the SIMPLE pressure-velocity correction loop [20]. For solving the linear systems of equations a preconditioned conjugate gradient schemes have been used for velocity, temperature and pressure. All, velocity, temperature, and pressure equations were solved to tolerances $10^{-8}$.

In spite of some compelling features of finite volume method there are some undesirable numerical effects (for example, artificial diffusion [21]) that are influenced by the low-order interpolation of the convection terms in governing equations. In order to overcome those undesirable numerical effects, the second-order accurate linear upwind differencing scheme [22] was used in the present study.

The rest of the paper is organized as follows. The necessary mathematical background and numerical details are presented in the continuation of Section 2, which is followed by the grid refinement, numerical accuracy assessment and validation study (Section 3). Following this analysis, the results are presented and subsequently discussed (Section 4). The main findings are summarized and conclusions are drawn in the final section of the paper. 


\subsection{Governing equations}

For the present study a steady-state laminar flow of an incompressible Power-law fluid is considered. For such a flow the governing equations (mass, momentum and energy conservation) take the following form:

$$
\begin{gathered}
\frac{\partial v_{i}}{\partial x_{i}}=0 \\
\rho v_{j} \frac{\partial v_{i}}{\partial x_{j}}-\frac{\partial}{\partial x_{j}}\left[\eta(\dot{\gamma}) \frac{\partial v_{i}}{\partial x_{j}}\right]=-\frac{\partial p}{\partial x_{i}}+\rho g \beta\left(T-T_{C}\right)+\frac{\partial}{\partial x_{j}}\left[\eta(\dot{\gamma}) \frac{\partial v_{j}}{\partial x_{i}}\right] \\
v_{j} \frac{\partial T}{\partial x_{j}}=\frac{\partial}{\partial x_{j}}\left[\alpha \frac{\partial v_{j}}{\partial x_{i}}\right]
\end{gathered}
$$

where the cold wall temperature $T_{C}$ is taken to be the reference temperature for evaluating the buoyancy term in the momentum conservation equation.

In momentum conservation equation (2) the constitutive (rheological) model is needed for the viscous function, which for the Power-law model takes the following form:

$$
\eta(\dot{\gamma})=K|\dot{\gamma}|^{n-1}
$$

where $K$ is the consistency parameter, $n$ is the flow index and $|\dot{\gamma}|=\sqrt{(1 / 2) \sum_{i} \sum_{j} \dot{\gamma}_{i j} \dot{\gamma}_{j i}}$ is the II. invariant of the symmetrical rate of deformation tensor with Cartesian components $\dot{\gamma}_{i j}=\left(\partial v_{i} / \partial x_{j}\right)+\left(\partial v_{j} / \partial x_{i}\right)$.

\subsection{Geometry and boundary conditions}

The simulation domain is shown schematically in Fig. 1, where the two vertical walls of a square cavity are maintained at different and constant temperatures $\left(T_{H}>T_{C}\right)$, whereas the other boundaries are considered to be adiabatic in nature. Both velocity components are identically zero on each boundary because of the no-slip condition and impenetrability of rigid boundaries. The adiabatic temperature boundary conditions for the horizontal insulated boundaries are given by $\partial T / \partial y=0$ at $y=0$ and $y=L$.
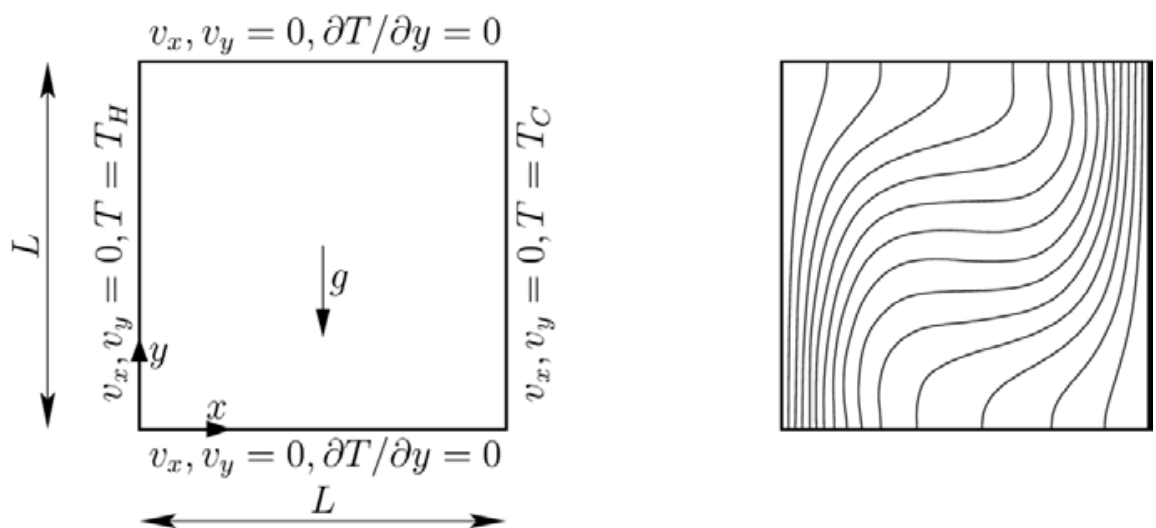

Figure 1: Schematic diagram of the simulation domain (left) and expected flow field (right).

In the present study, the heat transfer characteristics of Power-law fluids in a differentially heated square enclosure are analysed in terms of the mean Nusselt number:

$$
\overline{N u}=-\frac{1}{L} \int_{y=0}^{y=L}\left[\left.\frac{L}{T_{H}-T_{C}} \frac{\partial T}{\partial x}\right|_{x=0}\right] d y
$$


obtained for different values of $n$ with the same values of Rayleigh and Prandtl number [23].

$$
R a=\frac{g \beta \Delta T L^{2 n+1}}{\alpha^{n}(K / \rho)} ; \quad \operatorname{Pr}=\left(\frac{K}{\rho}\right) \alpha^{n-2} L^{2-2 n}
$$

\section{GRID REFINEMENT AND NUMERICAL ACCURACY STUDY}

The aim of most (if not all) numerical analysis is to achieve a certain accuracy with the smallest amount of a computational work. The difficulty to accomplish this usually lies in the unknown behaviour of the flow under numerical investigation. For that, the grid independence of the present results has been established on the basis of a detailed analysis of shear-thinning fluid $(n=0.50)$ at $R a=10000$ using three different non-uniform meshes (elements were concentrated towards each solid wall), the details of which are presented in Table II. The table includes the number of elements in a particular direction, as well as the normalized minimum cell size. With each grid refinement the number of elements in particular direction is increased and element size is reduced. Such a procedure is useful (and encountered in many numerical studies $[24,25]$ ) for obtaining a higher-order estimate of the flow value (value at infinite grid) from a series of lower-order discrete values [26].

Table II: Computational mesh characteristics.

\begin{tabular}{|c|c|c|c|}
\cline { 2 - 4 } \multicolumn{1}{c|}{} & Mesh $M I$ & Mesh $M I I$ & Mesh $M I I I$ \\
\hline$N_{x} \times N_{y}$ & $50 \times 50$ & $100 \times 100$ & $200 \times 200$ \\
\hline$\Delta_{\text {min }} / L$ & 0.002 & 0.001 & 0.0005 \\
\hline
\end{tabular}

For a general variable $\varphi$ the grid-converged value (i.e. extrapolated to the zero element size) according to the Richardson extrapolation is given as:

$$
\varphi_{\text {ext }}=\varphi_{\text {MIII }}-\left(\varphi_{M I I}-\varphi_{M I I I}\right) /\left(r^{p}-1\right)
$$

where $\varphi_{M I I I}$ is obtained on the finest grid and $\varphi_{M I I}$ is the solution based on next level of coarse grid, $r=2$ is ratio between the coarse to fine grid spacing and $p=2$ is the theoretical order of accuracy.

The numerical uncertainties for the mean Nusselt number and the maximum nondimensional vertical velocity on the horizontal mid-plane of the enclosure are presented in Table III.

Table III: Grid refinement and numerical accuracy study.

\begin{tabular}{|c|c|c|c|c|c|}
\cline { 2 - 6 } \multicolumn{1}{c|}{} & Mesh $M I$ & Mesh $M I I$ & Mesh MIII & $\varphi_{\text {ext }}$ & Error \\
\hline$\overline{N u}$ & 6.6848 & 6.7071 & 6.7129 & 6.7149 & $0.12 \%$ \\
\hline$v_{y, \max }^{*}$ & 125.4665 & 126.5854 & 126.8781 & 126.9757 & $0.31 \%$ \\
\hline
\end{tabular}

Results of calculations of the numerical accuracy (Table III) indicate that as the mesh is refined successively from $50^{2}$ to $200^{2}$ there is a consistent improvement in the accuracy of the predicted values, and the agreement between the predictions obtained with mesh MII and extrapolated values is extremely good for both flow variables under consideration (the discretisation error levels are smaller than $0.50 \%$ ). Based on this, the simulations in the remainder of the paper were conducted on mesh MII which provided a reasonable compromise between high accuracy and computational effort.

In addition to the grid-dependency study, the results for Newtonian fluid $(n=1.00)$ have also been compared against the numerical results of Turan et al. [27]. The comparisons 
(summarised in Table IV) between the present and corresponding benchmark values are very good and entirely consistent with our grid-dependency studies.

Table IV: Comparison of present results for Newtonian fluid with the benchmark [27] for $\operatorname{Pr}=7$.

\begin{tabular}{|c|c|c|}
\cline { 2 - 3 } \multicolumn{1}{c|}{} & $\begin{array}{c}R a=10^{3} \\
\overline{N u}\end{array}$ & $\begin{array}{c}R a=10^{4} \\
\overline{N u}\end{array}$ \\
\hline Present study & 1.117 & 2.272 \\
\hline Turan et al. [27] & 1.118 & 2.274 \\
\hline
\end{tabular}

\section{RESULTS AND DISCUSSION}

\subsection{Velocity and temperature distribution}

It is instructive to inspect the distributions of dimensionless temperature $\theta=\left(T-T_{C}\right) /\left(T_{H}-T_{C}\right)$ and dimensionless vertical velocity component $v_{y}^{*}=\left(v_{y} L\right) / \alpha$ in order to understand the effects of Power-law index on the heat transfer characteristics (i.e. variation of the mean Nusselt number $\overline{N u}$ ) during natural convection of Power-law fluids in the side heated square enclosure. The variations of $\theta$ and $v_{y}^{*}$ along the horizontal mid-plane $(y / L=0.50)$ are presented in Figs. 2 and 3 for different values of $n$. The distributions of dimensionless horizontal velocity component $v_{x}^{*}$ are not shown as the horizontal velocity component is of the same order of magnitude for square enclosure.
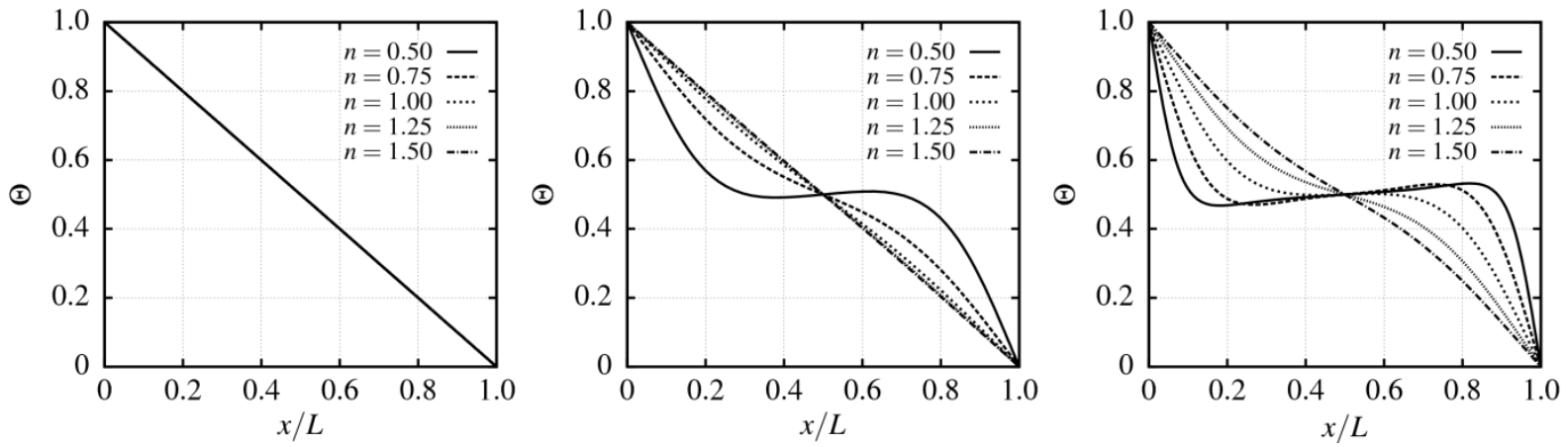

Figure 2: Variations of non-dimensional temperature $\theta$ for $R a=10^{1}$ (left), $R a=10^{3}$ (middle) and $R a=10^{4}$ (right).
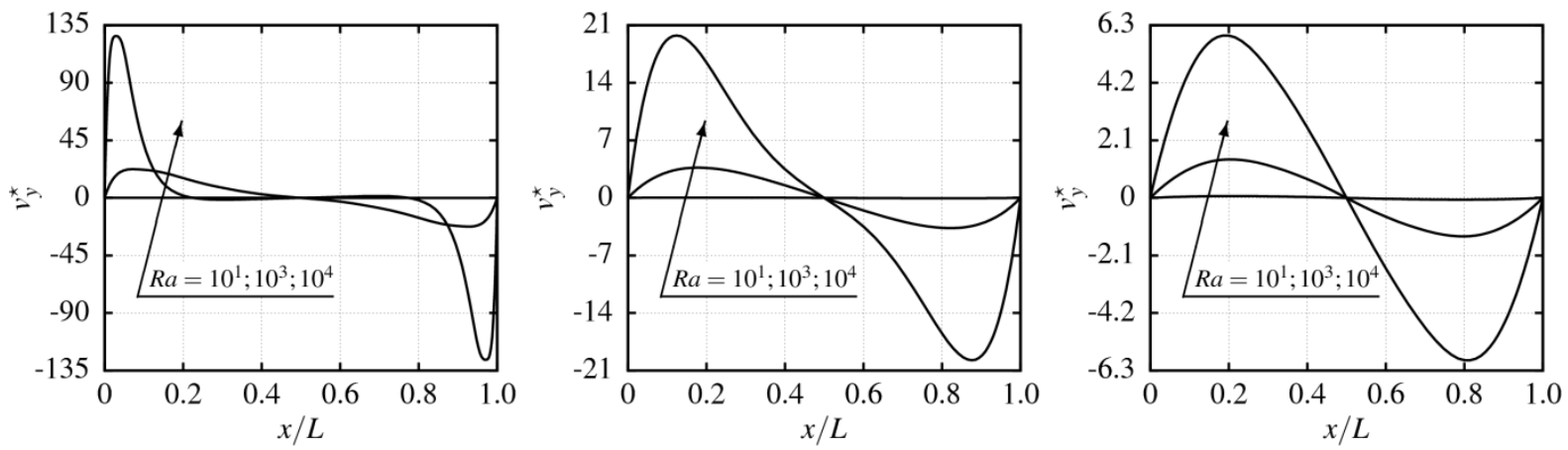

Figure 3: Variations of non-dimensional vertical velocity $v_{y}^{*}$ for $n=0.50$ (left), $n=1.00$ (middle) and $n=1.50$ (right).

It is evident from Fig. 2 that at lower values of Rayleigh number $\left(R a=10^{1}\right)$ the distribution of $\theta$ is completely linear and the vertical velocity component $v_{y}^{*}$ is essentially negligible due to very weak flow as the effects of buoyancy forces are dominated by viscous 
effects. Under this circumstance, the heat transfer takes place principally by conduction across the square enclosure. The effects of buoyancy force strengthen relative to the viscous force with increasing $R a$, which, in turn, augments the heat transfer by convection due to stronger buoyancy-driven flow with higher vertical velocity magnitude. This effect is clearly evident from Fig. 3, which indicates that the peak values of the vertical velocities increase due to the intensified convective activities with increase in Power-law fluid Rayleigh number. The steep rise in the $v_{y}^{*}$ velocity gradient in the near hot wall region also confirms the increased convective activity at higher $R a$ values as observed in Fig. 3.
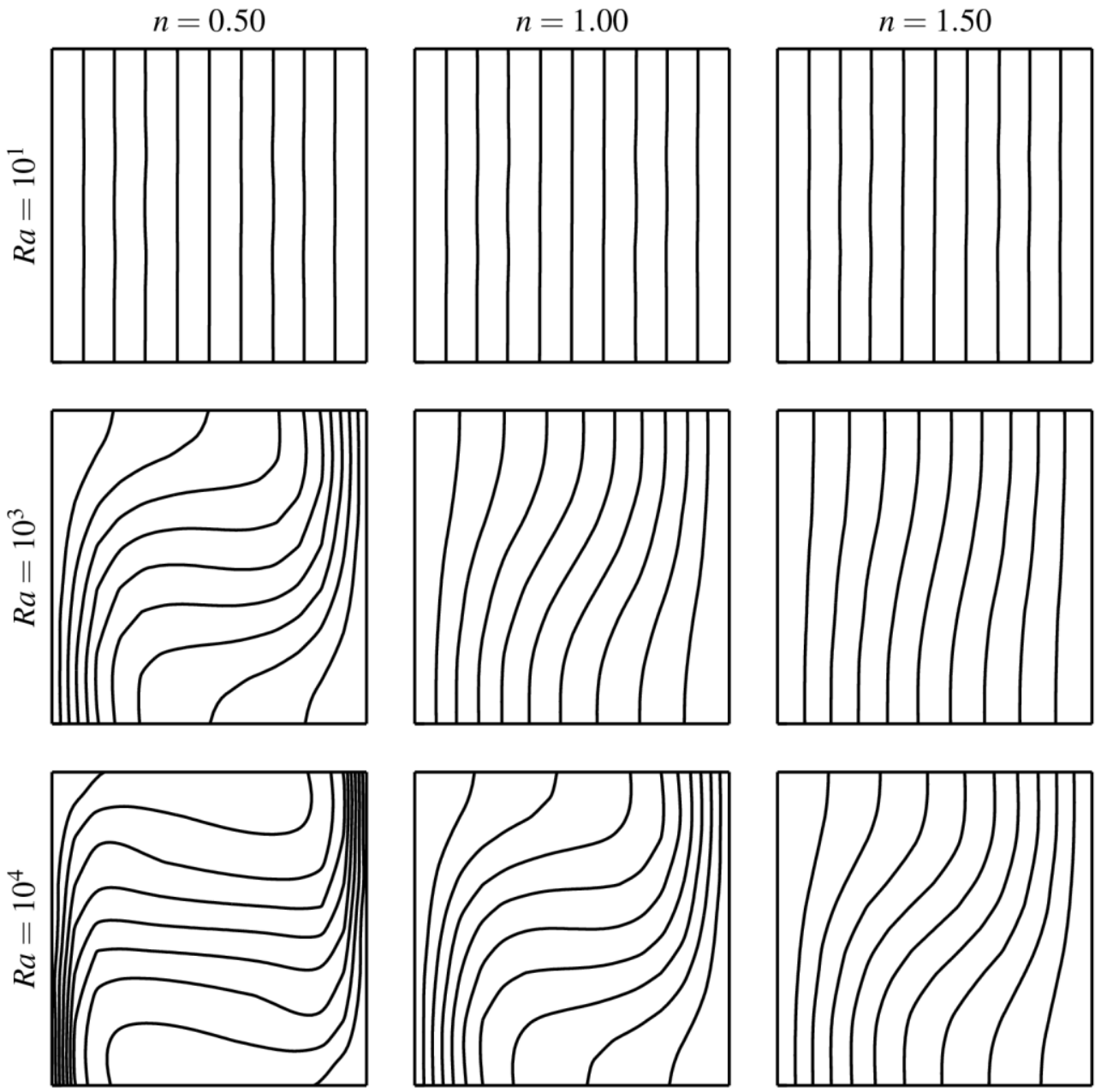

Figure 4: Isotherm contours.

Furthermore, it is evident from Fig. 2 that the distributions of $\theta$ become increasingly nonlinear as the value of Power-law index decreases, which suggests that the effects of convection become increasingly strong for decreasing values of $n$ when Rayleigh number is kept constant. This is further supported by the plot in Fig. 3 which shows that the magnitude of $v_{y}^{*}$ increases as the Power-law index decreases. This in turn means, that the strength of the buoyancy forces becomes increasingly strong in comparison to the viscous flow resistance for decreasing values of Power-law index and this effect is particularly effectual for the shearthinning Power-law fluids $(n<1.00)$. On the other hand, the effects of convection become increasingly weak in comparison to viscous forces with increasing for the shear-thickening Power-law fluids. These effects can be seen in smaller values of vertical velocity component and more linear distribution of dimensionless temperature for $n>1.00$. 
These findings are entirely consistent with earlier findings in the context of the natural convection of generalized Newtonian fluids [23, 27] as well as nanofluids [28, 29]. Furthermore, the scaling analysis [23] suggests that both hydrodynamic and thermal boundary layer thickness become progressively thin as the Power-law index value decreases when Rayleigh number is kept unaltered.

Moreover, the thinning of both hydrodynamic and thermal boundary layer thickness with decreasing $n$ can be further seen from the isotherms shown in Fig. 4. It can be observed from Fig. 4 that the isotherms become progressively more curved with decreasing value of Powerlaw index as a result of the strengthening of convective heat transfer transport.

\subsection{Mean Nusselt number}

Fig. 5 presents the variation of the mean Nusselt number along the heated wall with the Rayleigh number. For smaller values of the Rayleigh number convection in the Power-law fluid is extremely weak (practically negligible), and the thermal transport is principally conduction-driven, so the mean Nusselt number equals $\overline{N u}=1.00$ and its value is independent of the Rayleigh number value for both shear-thinning $(n<1.00)$ and shearthickening fluid $(n>1.00)$. As the Rayleigh number increases, the shear-thickening fluid remains in the conductive heat transfer regime, while convective thermal transport becomes of significant importance in the shear-thinning fluid.
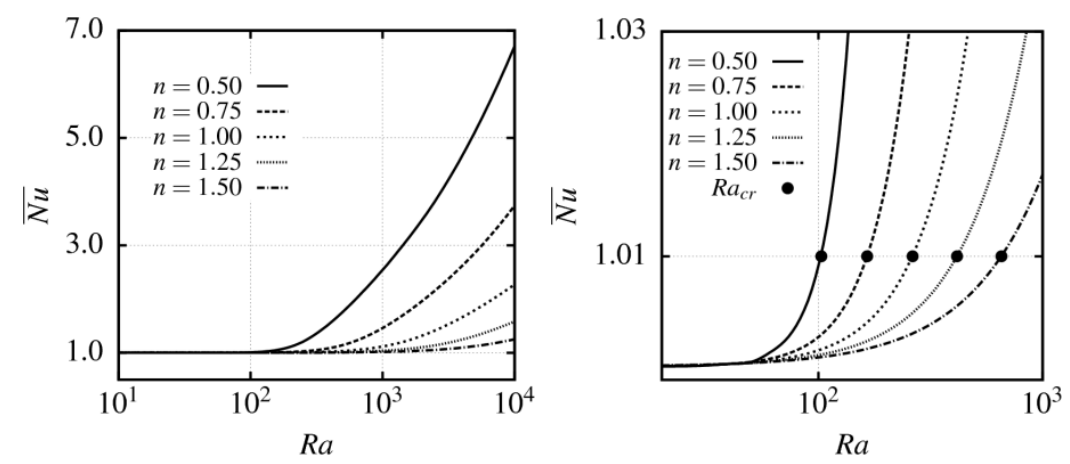

Figure 5: Variation of the mean Nusselt number with the Rayleigh number (left) and onset of the heat transfer convection (right).

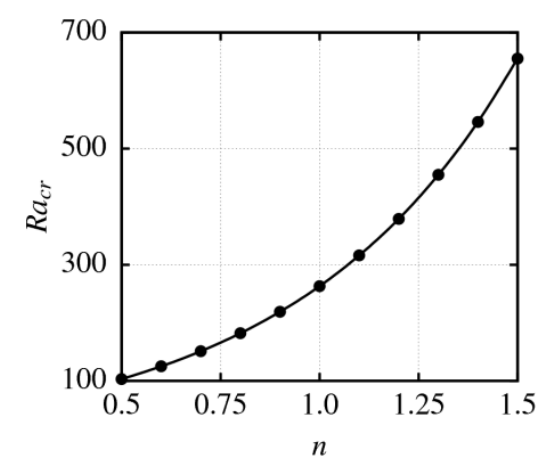

Figure 6: Variation of the critical Rayleigh number with the Power-law index.

The point above which the convective heat transfer starts to play an important role in the thermal transport (i.e. the critical value of the Rayleigh number at which the mean Nusselt number equals 1.01) depends on the value of the Power-law index. The higher is the value of the Power-law index, the more delayed is the onset of heat transfer convection (Fig. 6). When the Power-law fluid is in the convective heat-transfer regime $(\overline{N u} \geq 1.01)$, the mean Nusselt 
number is a monotonic increasing function of the Rayleigh number (Fig. 5) and attains lower values for the shear-thickening fluids $(n>1.00)$ at a given Rayleigh number.

One should note that a detailed analysis of the precise onset of fluid motion in this configuration is beyond the scope of the current analysis. In the present work, we are interested in identifying the point above which the convective heat transfer in Power-law fluids starts to play an important role in the thermal transport by using the criterion of $\overline{N u}>1.01$ (similar as in $[28,29]$ ), and this serves the main purpose of the current analysis.

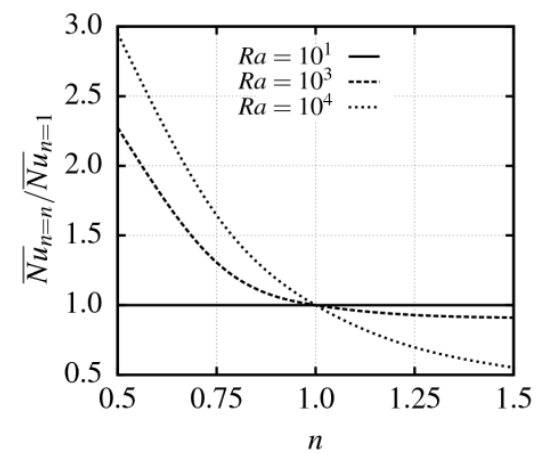

Figure 7: Variation of the normalised mean Nusselt number with the Power-law index.

Finally, Fig. 7 illustrates the variation of the normalised mean Nusselt number (i.e. the mean Nusselt number for each $n$ is normalised by the corresponding value for a Newtonian fluid) with the Power-law index; such a normalisation elucidates the role of the Power-law rheology in an unambiguous manner.

When the Power-law fluid is in the conduction dominated regime, the corresponding value of the normalised mean Nusselt number equals 1.00 and is independent of the Powerlaw index value. On the other hand, when the Power-law fluid is in the convection dominated regime, the variation in the overall heat transport for a non-Newtonian fluid is remarkable. Qualitatively, for the shear-thinning behaviour $(n<1.00)$, the heat transport increses with the increasing Rayleigh number and decreases for the shear-thickening behaviour $(n>1.00)$. Similar results were also observed in the numerical study of Kim et al. [15] for natural convection of the shear-thinning fluid in the range $10^{5} \leq R a \leq 10^{7}$ and $10^{2} \leq \operatorname{Pr} \leq 10^{4}$.

\section{CONCLUSION}

In the present study, the heat transfer characteristics of two-dimensional steady and laminar buoyancy driven flow of inelastic generalised Newtonian fluids in a square enclosure with differentially heated side walls subjected to constant temperatures have been studied by numerical means. The shear-thinning, Newtonian and shear-thickening viscous behaviour of a fluid has been considered with the well known and widely used Power-law model. The system of the mass, momentum and energy conservation equations has been solved by the open-source OpenFOAM CFD software package based on the SIMPLE algorithm. The effects of the Rayleigh number $R a$ and Power-law index $n$ at Prandtl number value $\operatorname{Pr}=10$ on heat and momentum characteristics in conduction and convection regime have been systematically investigated.

The influence of computational grid refinement on the present numerical predictions was studied throughout the examination of spatial (grid) convergence for the flow of shearthinning fluid $(n=0.50)$ at Rayleigh number value $R a=10^{4}$. In addition, Richardson's extrapolation technique was used to quantify the numerical accuracy for the mean Nusselt number and the maximum non-dimensional vertical velocity component. By utilizing extremely fine and mesh refinement towards each solid wall, the resulting discretisation error 
levels are well below $0.50 \%$. The numerical method was validated for the case of natural convection of Newtonian fluid at $\operatorname{Pr}=7$ which the results are available in the open literature. Remarkable agreement of present results with the benchmark results yields sufficient confidence in the present numerical procedure and results.

For a range of the Rayleigh number $10^{1} \leq R a \leq 10^{4}$ the conductive and convective buoyancy driven flow of the Power-law fluid was thoroughly examined with focus on the effects of shear-thinning and shear-thickening viscous behaviour on the flow features. Based on the present numerical results, the following important conclusions can be emphasised:

- For smaller values of the Rayleigh number $\left(R a<R a_{c r}\right)$ the heat convection in the Powerlaw fluid is practically negligible and the thermal transport is principally conductiondriven; therefore, the mean Nusselt number equals $\overline{N u}=1.00$ and its value is independent of the Rayleigh number value for both shear-thinning $(n<1.00)$ and shear-thickening fluid $(n>1.00)$.

- The point above which the convective heat transfer starts to play an important role in the thermal transport (i.e. the critical value of the Rayleigh number at which the mean Nusselt number equals 1.01) depends on the value of the Power-law index. The higher is the value of the Power-law index, the more delayed is the onset of heat transfer convection.

- In the convection dominated heat-transfer regime mean Nusselt number rises monotonically with increasing values of the Rayleigh number. However, mean Nusselt number (at the same value of the Rayleigh number) decreases as the Power law index increases.

- When the Power-law fluid is in the convection dominated regime $\left(R a>R a_{c r}\right)$, the variation in the overall heat transport for a Power-law fluid is remarkable. Qualitatively, for the shear-thinning behaviour $(n<1.00)$, the heat transport increses with the increasing Rayleigh number and decreases for the shear-thickening behaviour $(n>1.00)$.

\section{ACKNOWLEDGEMENT}

The authors are very much obliged to the very competent reviewers for their constructive and helpful comments and suggestions. These led to improvements in the revised paper.

\section{REFERENCES}

[1] Yayla, S. (2013). Flow characteristic of staggered multiple slotted tubes in the passage of a fin tube heat exchanger, Strojniski vestnik - Journal of Mechanical Engineering, Vol. 59, No. 7-8, 462-472, doi:10.5545/sv-jme.2012.902

[2] Jenko, M. (2015). Numerical cooking for pasteurized soft boiled eggs, Strojniski vestnik Journal of Mechanical Engineering, Article in Press, doi:10.5545/sv-jme.2014.2187

[3] Tadamalle, A. P.; Reddy, Y. P; Ramjee, E.; Reddy, V. K. (2014). Influence of welding speed on the melting efficiency of Nd:YAG laser welding, Advances in Production Engineering \& Management, Vol. 9, No. 3, 128-138, doi:10.14743/apem2014.3.182

[4] Andronache, C.; Socalici, A.; Hepuţ, T. (2013). The influence of micro coolers on the physicalmechanical characteristics of the steels used in making railway monoblock wheels, Technical Gazette, Vol. 20, No. 3, 419-423

[5] De Vahl Davis, G. (1983). Natural convection of air in a square cavity: A bench mark numerical solution, International Journal for Numerical Methods in Fluids, Vol. 3, No. 3, 249-264, doi: $10.1002 /$ fld. 1650030305

[6] Hortmann, M.; Perić, M.; Scheuerer, G. (1990). Finite volume multigrid prediction of laminar natural convection: Bench-mark solutions, International Journal for Numerical Methods in Fluids, Vol. 11, No. 2, 189-207, doi:10.1002/fld.1650110206 
[7] Nonino, C.; Croce, G. (1997). An equal-order velocity-pressure algorithm for incompressible thermal flows, Part 2: Validation, Numerical Heat Transfer, Part B: Fundamentals, Vol. 32, No. 1, 17-35, doi: $10.1080 / 10407799708914997$

[8] Buyruk, E.; Karabulut, K. (2013). Numerical investigation into heat transfer for threedimensional plate fin heat exchangers with fins placed perpendicular to flow, Transactions of FAMENA, Vol. 37, No. 2, 87-102

[9] Honus, S.; Juchelková, D. (2014). Mathematical models of combustion, convection and heat transfer in experimental thermic device and verification, Technical Gazette, Vol. 21, No. 1, 115122

[10] Hu, Y.-P.; Li, Y.-R.; Yuan, X.-F.; Wu, C.-M. (2013). Natural convection of cold water near its density maximum in an elliptical enclosure containing a coaxial cylinder, International Journal of Heat and Mass Transfer, Vol. 60, 170-179, doi:10.1016/j.ijheatmasstransfer.2012.12.059

[11] Saha, S. C.; Gu, Y. T. (2015). Natural convection in a triangular enclosure heated from below and non-uniformly cooled from top, International Journal of Heat and Mass Transfer, Vol. 80, 529-538, doi:10.1016/j.ijheatmasstransfer.2014.09.047

[12] Perko, L.; Friesenbichler, W.; Obendrauf, W.; Buchebner, V.; Chaloupka, G. (2013). Elongational viscosity of rubber compounds and improving corresponding models, Advances in Production Engineering \& Management, Vol. 8, No. 2, 126-133, doi:10.14743/apem2013.2.160

[13] Rossatto, V.; Picatonotto, T.; Vione, D.; Carlotti, M. E. (2003). Behavior of some rheological modifiers used in cosmetics under photocatalytic conditions, Journal of Dispersion Science and Technology, Vol. 24, No. 2, 259-271, doi:10.1081/DIS-120019977

[14] Chhabra, R. P.; Richardson, J. F. (2008). Non-Newtonian Flow and Applied Rheology, $2^{\text {nd }}$ edition, Butterworth-Heinemann, Oxford

[15] Kim, G. B.; Hyun, J. M.; Kwak, H. S. (2003). Transient buoyant convection of a power-law nonNewtonian fluid in an enclosure, International Journal of Heat and Mass Transfer, Vol. 46, No. 19, 3605-3617, doi:10.1016/S0017-9310(03)00149-2

[16] Lamsaadi, M.; Naimi, M.; Hasnaoui, M.; Mamou, M. (2006). Natural convection in a vertical rectangular cavity filled with a non-Newtonian power law fluid and subjected to a horizontal temperature gradient, Numerical Heat Transfer: Part A: Applications, Vol. 49, No. 10, 969-990, doi: $10.1080 / 10407780500324988$

[17] Lamsaadi, M.; Naimi, M.; Hasnaoui, M. (2006). Natural convection heat transfer in shallow horizontal rectangular enclosures uniformly heated from the side and filled with non-Newtonian power law fluids, Energy Conversion and Management, Vol. 47, No. 15-16, 2535-2551, doi:10.1016/j.enconman.2005.10.028

[18] Turan, O.; Sachdeva, A.; Chakraborty, N.; Poole, R. J. (2011). Laminar natural convection of power-law fluids in a square enclosure with differentially heated side walls subjected to constant temperatures, Journal of Non-Newtonian Fluid Mechanics, Vol. 166, No. 17-18, 1049-1063, doi:10.1016/j.jnnfm.2011.06.003

[19] Weller, H. G.; Tabor, G.; Jasak, H.; Fureby, C. (1998). A tensorial approach to computational continuum mechanics using object-oriented techniques, Computers in Physics, Vol. 12, 620-631, doi: $10.1063 / 1.168744$

[20] Patankar, S. V.; Spalding, D. B. (1972). A calculation procedure for heat, mass and momentum transfer in three-dimensional parabolic flows, International Journal of Heat and Mass Transfer, Vol. 15, No. 10, 1787-1806, doi:10.1016/0017-9310(72)90054-3

[21] Versteeg, H. K.; Malalasekera, W. (1995). An Introduction to Computational Fluid Dynamics: The Finite Volume Method, Addison Wesley Longman Ltd., Harlow

[22] Shyy, W.; Thakur, S.; Wright, J. (1992). Second-order upwind and central difference schemes for recirculating flow computation, American Institute of Aeronautics and Astronautics Journal, Vol. 30, No. 4, 923-932, doi: $10.2514 / 3.11010$

[23] Turan, O.; Sachdeva, A.; Poole, R. J.; Chakraborty, N. (2013). Aspect ratio and boundary conditions effects on laminar natural convection of power-law fluids in a rectangular enclosure with differentially heated side walls, International Journal of Heat and Mass Transfer, Vol. 60, 722-738, doi:10.1016/j.ijheatmasstransfer.2013.01.017 
[24] Bilus, I.; Morgut, M.; Nobile, E. (2013). Simulation of sheet and cloud cavitation with homogenous transport models, International Journal of Simulation Modelling, Vol. 12, No. 2, 94-106, doi:10.2507/IJSIMM12(2)3.229

[25] Ternik, P.; Rudolf, R. (2014). Laminar forced convection heat transfer characteristics from a heated cylinder in water based nanofluids, International Journal of Simulation Modelling, Vol. 13, No. 3, 312-322, doi:10.2507/IJSIMM13(3)5.271

[26] Roache, P. J. (1994). Perspective: A method for uniform reporting of grid refinement studies, Journal of Fluids Engineering, Vol. 116, No. 3, 405-413, doi:10.1115/1.2910291

[27] Turan, O.; Chakraborty, N.; Poole, R. J. (2010). Laminar natural convection of Bingham fluids in a square enclosure with differentially heated side walls, Journal of Non-Newtonian Fluid Mechanics, Vol. 165, No. 15-16, 901-913, doi:10.1016/j.jnnfm.2010.04.013

[28] Ternik, P.; Rudolf, R. (2014). Conduction and convection heat transfer characteristics of waterbased Au nanofluids in a square cavity with differentially heated side walls subjected to constant temperatures, Thermal Science, Vol. 18, Suppl. 1, S189-S200, doi:10.2298/TSCI130604082T

[29] Ternik, P.; Rudolf, R.; Žunič, Z. (2015). Heat-transfer characteristics of a non-Newtonian Au nanofluid in a cubical enclosure with differentially heated side walls, Materials and Technology, Vol. 49, No. 1, 87-93 